\title{
Erratum to: Sleep Disorders and Menopause
}

\author{
Youngsin Han, Jinju Lee, Hyun Hee Cho, Mee-Ran Kim
}

Department of Obstetrics and Gynecology, College of Medicine, The Catholic University of Korea, Seoul, Korea

J Menopausal Med 2019;25:83-7

https://doi.org/10.6118/jmm.19192

The authors regret that the order of Youngsin Han and Jinju Lee was unintentionally changed in the original version of this article.

The correct list of authors is:

Youngsin Han, Jinju Lee, Hyun Hee Cho, Mee-Ran Kim

The electronic version of this article has been updated at https://doi.org/10.6118/jmm.19192.

The Journal would like to assure readers that this does not affect any other content of the article.

Address for Correspondence: Mee-Ran Kim, Department of Obstetrics and Gynecology, College of Medicine, The Catholic University, 222 Banpodaero, Seocho-gu, Seoul 06591, Korea

Tel: 82-2-2258-6170, E-mail: mrkim@catholic.ac.kr, ORCID: https://orcid.org/0000-0003-4492-0768 